\title{
OUTCOME OF SEVERE RECEPTIVE LANGUAGE DISORDER
}

The clinical and familial characteristics of severe receptive specific language impairment (SLI) were studied in 58 affected children (ratio of boys to girls 2:1) at the Department of Child Life and Health, University of Edinburgh, Scotland. When tested at a mean of 6 years after school entry, patients diagnosed with SLI had a normal mean nonverbal IQ, but one third had a persistent SLI, only $2(3 \%)$ having attained normal scores on language tests. One third of siblings, previously considered normal, had abnormal language scores, and most family members had impaired phonological auditory memory, as measured by non-word repetition. A language learning difficulty occurred in $69 \%$ of first-degree relatives. Severe receptive SLI is almost always associated with severe reduction in expressive language skills. (Clark A, O'Hare A, Watson J et al. Severe receptive language disorder in childhood - familial aspects and long-term outcomes: results from a Scottish study. Arch Dis Child July 2007;92:614-619). (Respond: Professor Anne O'Hare, Department of Child Life and Health, 20 Sylvan Place, Edinburgh EH9 1UW, UK).

COMMENT. Receptive specific language impairment is characterized by difficulty in acquiring language comprehension despite normal non-verbal intelligence. Receptive language disorder has a poor prognosis and rarely resolves. Siblings and other family members are at increased risk of language impairments. Early diagnosis and intervention are recommended.

A follow-up study in later adult life, also at the University of Edinburgh, found that children with severe receptive developmental language disorder, reassessed in their midthirties, had deficits in verbal short-term memory and phonological processing, with social adaptation difficulties and increased risk of psychiatric disorder (Clegg $\mathbf{J}$ et al. J Child Psychol Psychiatry 2005;46:128-149).

Synthetic speech in treatment of language delay. Tallal P, at Rutgers University, and Merzenich MM et al, at University of California, San Francisco, reported remarkable and significant improvements in receptive speech and language comprehension in languagelearning impaired children following training with acoustically modified speech stimuli. (Tallal P et al. Science 1996;271:81-84). Brief, rapidly changing components of speech were prolonged and emphasized, and coupled with adaptive training exercises. When presented in slower forms and rates, stimuli were correctly perceived and receptive language improved. (Merzenich MM et al. Science 1996;271:77-81). Compared to natural speech training, acoustically modified speech training was significantly more effective.

\section{SEIZURE DISORDERS}

\section{EPILEPSY AND SPINOCEREBELLAR ATAXIA}

A large consanguinous family from Saudi Arabia with 4 affected children presenting with an autosomal recessive ataxia, generalized tonic-clonic epilepsy and mental retardation is reported from the Institut de Genetique, Universite Louis Pasteur, Illkirch, France; Division of Pediatric Neurology, King Saud University, Riyadh, Saudi Arabia; and other centers. None of the 4 had myoclonus or mental deterioration. MRI of one patient revealed 
posterior white matter hyperintensities and mild cerebellar vermis atrophy, and the muscle biopsy showed vacuolization of the sarcotubular system. EEG was normal in 2 patients and showed epileptiform discharges in 2, generalized in one and bioccipital in another. The defective gene was localized to a new disease locus on chromosome 16q21-q23. Epilepsy presented at age 9-12 months, and ataxia was noted when the children started to walk at 2-3 years. All 4 had psychomotor delay and learning disabilities. Deep tendon reflexes were diminished, plantar responses were equivocal, speech was dysarthric, and the eye exam showed nystagmus. (Gribaa M, Salih M, Anheim M et al. A new form of childhood onset, autosomal recessive spinocerebellar ataxia and epilepsy is localized at 16q21-q23. Brain July 2007;130:1921-1928). (Respond: Dr M Koenig, Institut de Genetique et de Biologie Moleculaire et Cellulaire, 1 rue Laurent Fries BP10142, 67404 Illkirch cedex, France).

COMMENT. The differential diagnosis of recessive spinocerebellar ataxia with progressive myoclonus epilepsy and/or generalized tonic-clonic seizures includes UnverrichtLundborg disease, Lafora disease, neuronal ceroid lipofuscinoses, sialidoses, the sensory ataxia, neuropathy, dysarthria and ophthalmoparesis (SANDO) syndrome, and myoclonic epilepsy with ragged red fibers (MERRF) syndrome. Age at onset, absence of myoclonus and dementia, and EEG and MRI findings allowed exclusion of these disorders and the definition of a new cpilepsy/ataxia syndrome localized to the 16q21-q23 locus.

\section{DIAGNOSTIC INACCURACIES IN CHILDREN WITH "FIRST SEIZURE"}

A prospective cohort study of 127 children aged 1 month through 17 years seen in the First Seizure clinic at the Alberta Children's Hospital between Jan 1, 2004 and August 30, 2005 determined the range of diagnoses and the prevalence of previous unrecognized seizures. The diagnosis was epileptic in 94 (74\%), nonepileptic in $31(24 \%)$ and unclassified in two (2\%). Pediatricians referred true epileptic events in $92 \%$ cases, ED physicians $76 \%$, and family physicians $65 \%$. Mean age at presentation was 8 years. Development was delayed in $15 \%$; the neurologic examination was abnormal in $11 \%$. True epileptic seizures were generalized in $32(34 \%)$ and partial in $62(66 \%)$. Fifteen $(16 \%)$ had an epilepsy syndrome. Over a 1-year follow-up period, $42(45 \%)$ children presenting with an epileptic seizure were diagnosed with epilepsy (recurrent seizures). A prior probable seizure in $38 \%$ was recognized by the referring physician in only one case. Unrecognized events included: absence seizure (2), myoclonic (5), and partial complex (8). An EEG obtained in all children with seizures was abnormal in $41 \%$. EEGs obtained early ( $<48$ hours) showed abnormalities in $47 \%$ compared to $44 \%$ of those obtained late ( $>48$ hours), with no significant difference. (Hamiwka LD, Singh N, Niosi J, Wirrell EC. Diagnostic inaccuracy in children referred with "first seizure": Role for a First Seizure clinic. Epilepsia June 2007;48:1062-1066). (Reprints: Dr Lorie Hamiwka, Alberta Children's Hospital, Division of Pediatric Neurology, 1820 Richmond RD SW, Calgary, AB T2T 5C7, Canada).

COMMENT. Diagnostic inaccuracies in children with "first seizures" are common in general practice, one quarter of patients incorrectly diagnosed has having a seizure rather than a nonepileptic event, while a diagnosis of epilepsy is missed in over one-third. The authors recommend that children with a first seizure should be seen by a neurologist. 